\section{INTERNATIONAL DONATION CAMPAIGN}

The Pink Ribbon has been chosen to symbolise a joint international donation campaign by Hu-Friedy and Henry Schein. For each selected EverEdge instrument which is sold, Hu-Friedy will donate one Euro to the German Breast Cancer Initiative.

Coated with a special stainless steel alloy, Hu-Friedy's EverEdge high-precision instruments are extremely durable and retain their sharpness for much longer. The finely honed tips guarantee efficient scaling.

EverEdge technology combines state-of-the-art metallurgy with the latest heat treatment and cryogenic processing techniques. The result of this manufacturing method is a steel that is extremely hard and durable. This in turn ensures excellent wear

\section{DISPOSABLE IRRIGATION LINES}

Created with practitioners for practitioners, the Bien-Air Chiropro L is an ultra-efficient, versatile implantology system. It has been designed with efficiency and comfort in mind as the intuitive interface can be managed by the control pedal.

The patented peristaltic pump with disposable irrigation lines and insertion support ensures easy handling and optimum infection control. It includes the MX-LED self-ventilating motor, which offers LED lighting at equal intensity at both high and low speeds.

Incorporating seven of the leading brands of implants with their complete sequences, the Chiropro L can also be customised to your individual requirements. Featuring an internal irrigation system the 20:1 contraangle handpiece incorporates the

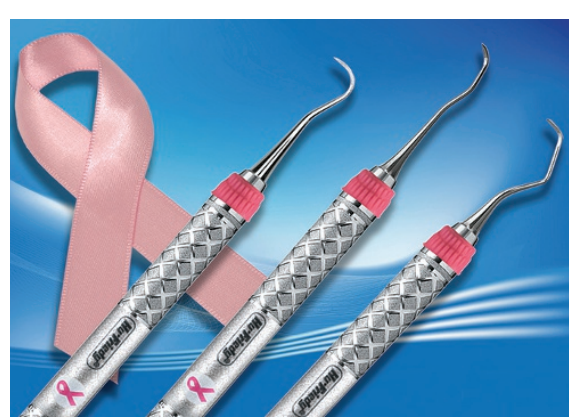

resistance and a long service life.

The coating covers the entire surface of the tip and allows the scalers to be re-sharpened over and over again. Unlike scaling and curette tips made of conventional steel, the finely honed EverEdge tips and cutting edges retain their sharpness for much longer and require less frequent re-sharpening.

\section{Reader response number $\mathbf{5 8}$}

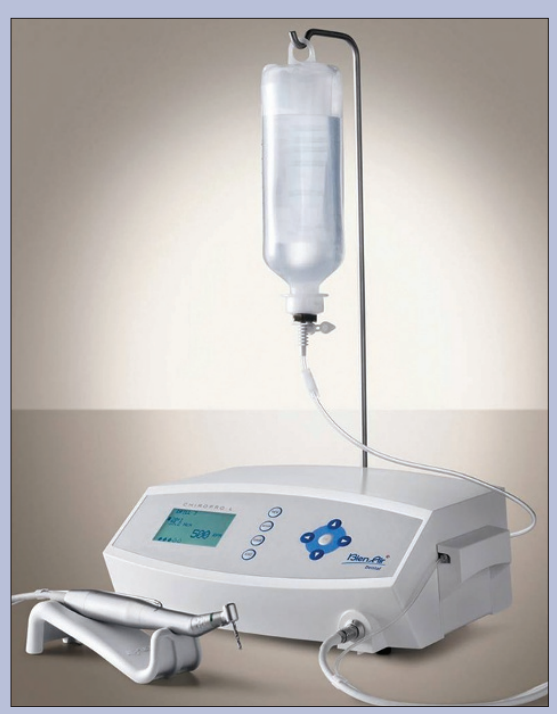

smallest head on the market allowing access to the tightest of areas.

Fitted with an exclusive double optical glass rod system, the handpiece provides uniform lighting of the operative field.

Reader response number 59

\section{OPTIMUM QUALITY DIGITAL IMAGES}

PracticeWorks has introduced the Kodak RVG 6500 to the market, the latest in its line of intraoral X-ray sensors. Developed to provide optimum quality digital images and improve image portability, the RVG 6500 enables adaptable positioning and connectivity. Features include wireless freedom with a portable, wireless Kodak RVG sensor utilising state-of-the-art WiFi technology (image upload time $>3 \mathrm{~s}$ ), and an intelligent positioning system which streamlines workflow.

The technology enables proper sensor alignment and angulation whilst IPS controls X-ray and $\mathrm{x}-\mathrm{y}$ beam placement to prevent distortions. It has a high image resolution and improved integration, as its compact, lightweight design features eliminate cables and fit any practice décor. An enhanced computer interface system and specific FMS interface also provide improved productivity.

The Kodak RVG 6500 is robust and flexible, compatible with the majority of X-ray generators and its WiFi technology even enables digital images to be transferred to an iPod.

Reader response number 61

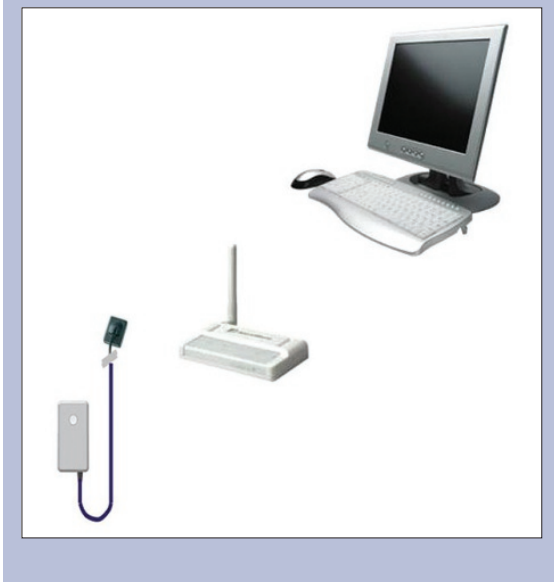

This lightweight, ergonomically designed, cordless curing light can be set to a range of practical curing times, including five, ten and 20-second intervals, as well as continuous mode to suit your individual requirements.

Reader response number 60 